\title{
Idade da menarca em escolares de uma comunidade rural do Sudeste do Brasil
}

\author{
Age at menarche among schoolgirls \\ from a rural community in Southeast Brazil
}

Carlos Henrique Falcão Tavares 1

Leris Salete Bonfanti Haeffner 1

Marco Antonio Barbieri 1

Heloisa Bettiol 1

Marisa Ramos Barbieri 2

Luiz de Souza 3

\footnotetext{
1 Departamento de Puericultura e Pediatria, Faculdade de Medicina de Ribeirão Preto,

Universidade de São Paulo. Av. Bandei rantes 3900, Ribeirão Preto, SP 14049-900, Brasil. hbettiol@fmrp.usp.br 2 Laboratório de Ensino de Ciências, Faculdade de Filosofia, Ciências e Letras de Ribei rão Preto, Universidade de São Paulo. Av. Bandei rantes 3900 Ribei rão Preto, SP 14040-901, Brasil. 3 Instituto de Saúde Coletiva, Universidade Federal de Mato Grosso. Av. Fernando Correa da Costa s/no, Cuiabá, MT 78068-900, Brasil.
}

Abstract The purpose of this study was to determi ne the 3 rd percentile $\left(P_{3}\right), 50$ th percentile $\left(P_{50}=\right.$ median age at menarche $\left.=M A M\right)$, and amplitude between the extremes $\left(P_{97}\right.$ and $\left.P_{3}\right)$ of age at menarche among schoolgirls in the county of Barrinha, São Paulo, Brazil. Values were correlated with socioeconomic conditions such as social class, number of siblings, and father's employment status. This was a cross-sectional study based on the use of status quo adjusted by logit for calculation of percentiles. A questionnaire was applied to 1,602 school girls aged 8 to 17 years (incomplete). MAM was 12 years $(\mathrm{y})$ and 6 months $(\mathrm{m})$, with a $\mathrm{P}_{97}$ of $10 \mathrm{y}$ and $2 \mathrm{~m}$ and a $\mathrm{P}_{3}$ of 14 $y$ and $10 \mathrm{~m}$. Girls from lower-income families and those with unemployed fathers showed later MAM. No difference in MAM was observed with respect to number of siblings. Amplitude between $\mathrm{P}_{97}$ ad $\mathrm{P}_{3}$ was great in the overall sample. We conclude that Barrinha presented a MAM similar to and even lower than regional values for Brazil and for some developed countries. The study of the interval between extreme percentiles proved to be a better indicator of biol ogical diversity and socioeconomic inequality than MAM al one.

Key words Menarche; Students; Adolescence; Rural Health

Resumo O objetivo deste estudo foi determinar os val ores dos percentis $3\left(P_{3}\right), 50\left(P_{50}=\right.$ Idade Mediana da Menarca $=I M M)$ e $97\left(P_{97}\right)$, e a amplitude entre os extremos $\left(P_{97}\right.$ e $\left.P_{3}\right)$, nas escol ares do Município de Barrinha, São Paulo, Brasil. Esses valores foram correlacionados com al gumas condi ções sóci o-econômi cas: classe social, número de irmãos e si tuação de desemprego do pai . Trata-se de estudo transversal, uti lizando o método "status quo", ajustado pel o Logi to para o cálculo dos percentis. Questionário foi o instrumento aplicado a 1.602 escolares entre 8 e 17 anos incompletos. A I MM foi 12 anos(a) e 6 meses $(\mathrm{m}), \mathrm{P}_{97}$ de $10 \mathrm{a} 2 \mathrm{~m}$ e $\mathrm{P}_{3}$ de $14 \mathrm{a} 10 \mathrm{~m}$. As meninas da classe social menos favorecida, e aquel as que referiram desemprego paterno apresentaram I M M mais tardia. Não houve diferença da IMM relacionada ao número de irmãos. A amplitude entre $\mathrm{P}_{97}$ e $\mathrm{P}_{3}$ mostrou-se ampla na amostra geral. Concluiu-se que Barrinha apresentou IMM semeIhante e até abaixo de valores regionais, do Brasil e de al guns países desenvol vi dos. O estudo do intervalo entre os percentis extremos mostrou ser um melhor indicador das diversi dades biológicas e das desigual dades sócio-econômi cas, do que a IMM isoladamente. Palavras-chave Menarca; Estudantes; Adolescência; Saúde Rural 
Introdução

A menarca, primeira menstruação, é um indicador de maturação no desenvolvimento da mulher. A menarca é o mais claro indicador de maturação sexual nas mulheres (Eveleth, 1998). O momento em que ela ocorre, idade da menarca (IM), é um indicador muito sensível de estresse ambiental e é também subordinada a significante controle genético (Cameron, 1998). Em estudos populacionais pode-se usar a idade mediana da menarca (IM M) (Healy, 1986).

Estudos têm demonstrado relações da IM com fatores genéticos (familiares, étnicos), com fatores geográficos como clima e altitude, com a sazonalidade, o estado nutricional, a atividade física, tensão emocional e efeito feromonal (Burger \& Gochfeld, 1985; Marshall \& Tanner, 1986; Tanner, 1989; Greksa, 1990; Wellens et al., 1990; Valenzuela et al., 1991; Ulijaszek et al., 1991; Veronesi \& Gueresi, 1994; Graber et al., 1995; Cameron \& Nagdee, 1996; Herman-Giddens et al., 1997).

Outros estudos encontraram IM M mais tardia na áreas rurais (Jordán, 1984; Ohsawa et al., 1997). No Brasil, dados da Pesquisa Nacional sobre Saúde e Nutrição mostram diferenças e associação positiva entre IM mais tardia e baixas condições sociais, comparando as macro regiões do Brasil, como também entre Brasil rural e urbano (Picanço, 1989). Entre meninas escolares em Ribeirão Preto, São Paulo, a IMM foi mais tardia nas do grupo rural do que no urbano, e neste último, foi mais tardia no grupo que habitava bairros de piores condições sócioeconômicas; a IMM foi mais precoce nas meninas com menos de dois irmãos (Fuzii, 1989), resultados semelhantes aos de outros trabaIhos (Ulijaszek et al., 1991; Cameron \& Nagdee, 1996).

A IM pode ser vista como um dos exemplos de síntese da interação do ser humano com o meio ambiente, na medida em que um e outro podem interagir mutuamente. O meio ambiente pode, por intermédio de vários fatores, alterar a IM M, e por outro lado a IMM (ficando mais precoce numa dada população) pode propiciar modificações no meio ambiente, desde novas mudanças educacionais até em termos de programas de saúde (Van Wieringen, 1986).

Neste trabalho foram determinados os valores dos percentis $3\left(P_{3}\right), 50\left(P_{50}=I M M\right)$ e 97 $\left(P_{97}\right)$, e a amplitude entre os extremos $\left(P_{97} \mathrm{e}\right.$ $\mathrm{P}_{3}$ ), da idade da menarca nas escolares do Município de Barrinha, São Paulo, Brasil. Esses vaIores foram correlacionados com al gumas condições sócio-econômicas: classe social, número de irmãos e situação de desemprego do pai.

\section{Casuística e métodos}

O Município de Barrinha pertence à região geoeconômica de Ribeirão Preto, Estado de São Paulo, na região Sudeste do Brasil. Conta com aproximadamente 5.500 moradias e foi estimada uma população de 23.604 habitantes para 1998, com base em dados do IBGE (1996). É comum o aumento de $10 \%$ da população na época de maior atividade da lavoura de cana de açúcar (abril a novembro), principal atividade econômica do município. Essas características sócio-geográficas permitem identificar Barrinha como cidade rural.

Trata-se de estudo epidemiológico do tipo transversal, cuja população alvo foram as escolares do sexo feminino que tinham entre 8 e 17 anos incompletos (96 a 203 meses) de idade, recrutadas entre as 2 as séries do primeiro grau até as das 3as séries do segundo grau, nas escolas do município. Em Barrinha todas as escolas são públicas, sendo uma municipal e quatro estaduais.

O método escolhido para o cálculo dos percentis da IM foi o "status quo", um modelo transversal (Mac Mahon, 1973). Este método tem a vantagem de ser mais rápido que o método prospectivo, pois não depende de avaliações em vários intervalos de tempo e, em relação ao recordatório (recall - method), é menos sujeito a erros, já que não depende da memória da informante.

A variável idade foi calculada tomando-se a data de nascimento e a data da entrevista, sendo a faixa etária dividida em intervalos de classe de um ano. Para cada intervalo de classe foi computada a freqüência de menarca "sim" e "não", ou seja, o número de meninas que referiam ter tido ou não a primeira menstruação, até o dia da entrevista. No cálculo da idade mediana da menarca, o ajuste da curva sigmóide obtida da distribuição percentual de menarca "sim" foi feito pelo método do Logito [log (proporção de menarca presente/ 1 - proporção de menarca presente)], obtendo-se uma reta. Com base na equação da reta foram calculadas a IMM e os percentis $\mathrm{P}_{97}$ e $\mathrm{P}_{3}$, aplicando-se a transformação antilogarítmica (Finney, 1964). A IMM (percentil 50) representou a idade, em anos e décimos de anos, em que $50 \%$ das meninas não haviam tido a menarca. O percentil 97 foi a idade em que $97 \%$ das meninas não haviam ainda tido a menarca; o percentil 3 significou a idade em que $3 \%$ não haviam tido a menarca. Foi usado o teste de Qui-quadrado $\left(\chi^{2}\right)$ para avaliação do ajustamento da distribuição logística, que mostrou um bom ajustamento em todos os casos. 
O instrumento metodológico escolhido para coleta das informações foi o questionário, por ter as vantagens de ser econômico, ágil, podendo ser administrado simultaneamente a um grande número de pessoas (Thiollent, 1988). O questionário foi auto-aplicado, com o consentimento e sem a identificação da respondente. Foi também enviada uma "Carta aos Pais" contendo informações sobre a pesquisa e solicitando a permissão para que as estudantes (filhas) respondessem ao questionário. O projeto foi aprovado pelo Comitê de Ética em Pesquisa do Hospital das Clínicas da Faculdade de Medicina de Ribeirão Preto da Universidade de São Paulo (HC/FMRP/USP).

Os contatos com o Secretário Municipal de Educação, com as Diretoras das Escolas, com os pais das alunas, além da busca dos dados sobre as escolares do município foram feitos com o auxílio de cinco professoras das escolas de Barrinha. Após a autorização conseguida em todas as instâncias acima foi realizada a aplicação dos questionários.

No questionário constavam dados referentes à idade da menina, se habitava zona urbana ou rural, se já havia tido a menarca; informações sobre tamanho e composição da família, condições de moradia e situação de desemprego dos pais. A classificação social foi feita de acordo com a classificação de Olsen \& Frische (1973), baseada na International Standard Classification of Occupations (1970) e modificada por Bettiol et al. (1998), que leva em conta a ocupação do chefe da família, sendo definidas quatro frações de classe social: 1) gerentes, executivos e empresários; 2) administradores e empresários de nível médio; 3) trabalhadores qualificados e semiqualificados; 4) trabal hadores não qualificados, estudantes e desempregados.

Da população de 2.458 crianças do sexo feminino estimada para Barrinha na faixa etária de 8 a 17 anos incompletos em 1998 (IBGE, 1996), 2.013 (81,9\%) freqüentavam as escolas do município. Destas, 317 (15,7\%) não responderam ao questionário por motivos diversos evasão escolar, falta no dia da aplicação do questionário, recusa dos pais. Dos 1.696 questionários respondidos, 94 (5,5\%) foram eliminados por erros no seu preenchimento, restando 1.602 questionários válidos para o estudo, correspondendo a $79,6 \%$ das meninas escolares nessa faixa etária. Em todas as idades o percentual de meninas estudadas representou mais de $50 \%$ da população daquela faixa etária.

Com os questionários respondidos, passouse a digitação e revisão dentro do programa Epi Info 6.02. Os dados foram trabalhados nos programas Epi Info 6.02, Stata 5.0 e SAS.

\section{Resultados}

A IMM foi de 12,52 anos ( 12 anos e 6 meses), que corresponde ao percentil 50, obtida na $\mathrm{Fi}-$ gura 1, valendo-se da curva sigmóide derivada da distribuição percentual de menarca presente segundo a faixa etária das escolares (Tabela 1). O percentil 3 correspondeu a 14,86 anos (14 anos e 10 meses), e o percentil 97, a 10,17 anos (10 anos e 2 meses). Nenhuma menina teve a menarca antes dos 9 nem após os 16 anos.

Figura 1

Representação da reta ajustada aos dados transformados (logitos) de menarca (presente) segundo a faixa etária nas escolares do Município de Barrinha, 1998.

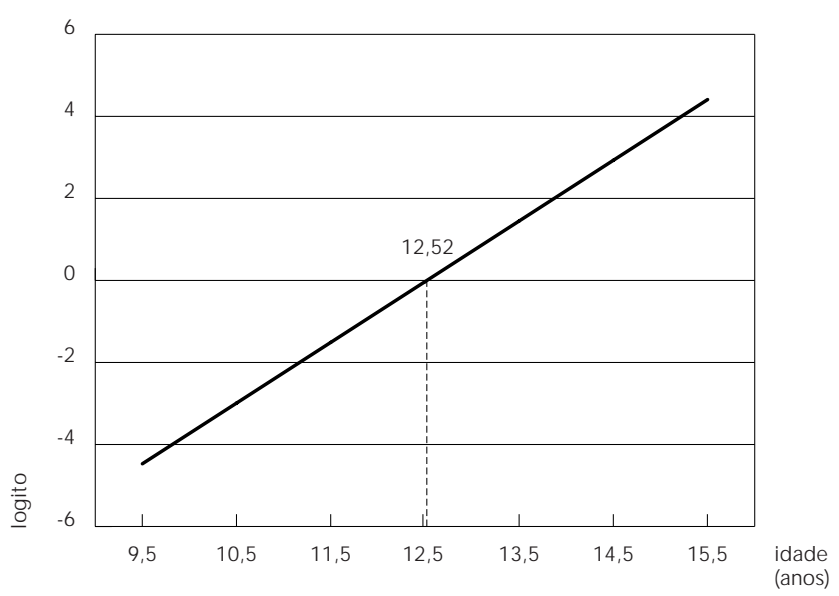

Tabela 1

Distribuição do percentual da menarca presente segundo a faixa etária das escolares no Município de Barrinha, 1998.

\begin{tabular}{lrrr}
\hline Idade & $\mathrm{n}$ & $\mathrm{R}$ & $\%$ \\
\hline $8,0-9,0$ & 146 & 0 & 0,00 \\
$9,0-10,0$ & 175 & 2 & 1,14 \\
$10,0-11,0$ & 231 & 12 & 5,19 \\
$11,0-12,0$ & 210 & 42 & 20,00 \\
$12,0-13,0$ & 209 & 106 & 50,71 \\
$13,0-14,0$ & 180 & 133 & 73,88 \\
$14,0-15,0$ & 191 & 178 & 93,19 \\
$15,0-16,0$ & 132 & 131 & 99,24 \\
$16,0-17,0$ & 128 & 128 & 100,00 \\
Total & 1.602 & 732 & \\
\hline
\end{tabular}

$\mathrm{n}=\mathrm{N}$ úmero de meninas entrevistadas.

$\mathrm{R}=\mathrm{N}$ úmero de meninas com menarca presente. 
Foram estabelecidos a IM M e os percentis 3 e 97 conforme as seguintes variáveis sócio-econômicas: classe social, número de irmãos e situação de desemprego dos pais (Tabela 2). Em relação à classe social, foram excluídos 275 questionários não classificados, que incluíram respostas não adequadas e/ ou aposentados. As escolares da classe social 1 - dos gerentes, executivos e empresários (em número de duas) - e as da classe social 2 - dos administradores e empresários de nível médio (em número de 25) - foram realocadas na classe social 3 - dos trabalhadores qualificados e semiqualificados por serem em pequeno número. Verifica-se que meninas pertencentes à classe social menos favorecida e aquelas cujos pais estavam em situação de desemprego apresentaram IMM mais tardia. Não foi observada diferença da IMM relacionada ao número de irmãos.

$A$ variação entre os percentis extremos - $P_{3}$ e $\mathrm{P}_{97}$ - foi ampla na amostra geral, sendo ainda mais ampla na maioria das variáveis sócio-econômicas estudadas, com exceção da situação de desemprego do pai. E todas, sem exceção, apresentaram $\mathrm{P}_{3}$ acima do $\mathrm{P}_{3}$ da amostra geral (Figura 2).

\section{Discussão}

Este estudo mostrou a IMM entre as escolares de Barrinha de 12,52 anos, sendo esse valor mais tardio nas meninas pertencentes à classe social menos favorecida e naquelas cujos pais estavam desempregados. Por outro lado, não

\section{Tabela 2}

Idade mediana da menarca (anos) segundo variáveis demográficas e sociais das escolares no Município de Barrinha, 1998.

\begin{tabular}{ll}
\hline Variáveis & Idade Mediana da Menarca \\
\hline Geral & 12,52 (12 anos e 6 meses) \\
Classe Social & \\
3 & 12,55 (12 anos e 7 meses) \\
4 & 12,63 (12 anos e 8 meses) \\
Situação de desemprego do pai & \\
Com desemprego & 12,73 (12 anos e 9 meses) \\
Sem desemprego & 12,44 (12 anos e 5 meses) \\
No de irmãos & \\
$<2$ & 12,51 (12 anos e 6 meses) \\
$\geq 2$ & 12,51 (12 anos e 6 meses) \\
\hline
\end{tabular}

houve diferença na IM M segundo o número de irmãos. A amplitude de variação entre os percentis extremos foi maior entre as classes sociais e entre meninas com pais em situação de desemprego do que na amostra geral de escolares.

Aproximadamente $80 \%$ das escolares de Barrinha participaram do estudo. Essas crianças representaram aproximadamente $65,2 \%$ da população feminina do município entre 8 e 17 anos incompletos. Embora esses números sejam expressivos, já que em algumas faixas etárias (10 e 11 anos) o número das escolares do trabal ho chegou a representar mais de $80 \%$ da população feminina da cidade, os resultados representam a população das escolares e não a população da cidade nessa faixa etária, uma vez que as características não conhecidas das meninas que não foram avaliadas poderiam modificar significativamente os resultados se elas tivessem participado do estudo.

A IMM nas escolares em Barrinha foi menor que a observada em países desenvolvidos como Bélgica, cerca de 13 anos (Vercauteren \& Suzanne, 1985), Inglaterra, 13,1 anos (Roberts \& Dann, 1975), Dinamarca e países nórdicos, 13 anos (Helm \& Gronlund, 1998). Também foi menor que a do Brasil em geral igual a 13,02 anos (Picanço, 1989), e que da própria cidade sede da região, Ribeirão Preto, com 12,62 anos (Fuzii,1989). Foi semelhante a de São Paulo Capital, 12,5 anos (Colli et al., 1985). Este resultado parece incompatível, comparando-se as condições sócio-ecônomicas de Barrinha com as de Ribeirão Preto, São Paulo e países desenvolvidos. Um dado que poderia explicar parcialmente esse achado é que as informações de I M M dessas localidades são de décadas passadas, com a possibilidade da interferência da tendência secular que, antecipando a menarca em 0,25 a 0,33 anos por década (Marshall \& Tanner, 1986; Cameron \& Nagdee, 1996), favoreceria o resultado de IM M mais precoce em Barrinha. Se a comparação for com países desenvolvidos como a Dinamarca, que está com a tendência secular estacionada e com a IM M em torno dos 13 anos (Helm \& Gronlund, 1998), uma explicação seria a possível interetnia presente em Barrinha, como no restante da população brasileira, com a presença de descendentes de africanos e asiáticos, que geralmente menstruam mais cedo que as meninas descendentes de europeus ( M arshall \& Tanner, 1986; Ulijaszek et al., 1991).

O fato de Barrinha ser uma cidade de características rurais levaria a uma expectativa de IM M mais tardia (Fuzii, 1989; Picanço, 1989; Liestol \& Rosemberg, 1995). Um dos motivos para a IMM em Barrinha ter sido mais precoce 
que as de Cuba e do Brasil (Geral, Urbano e Rural) seria a amostra em Barrinha ter sido colhida entre escolares e, nos outros estudos, na população geral. Jordán (1984) mostrou IM M mais precoce nas escolares quando comparadas com a de estudos realizados em meninas da população geral.

Foi comparado o intervalo (dispersão) entre os percentis 97 e 3 da IMM em Barrinha com dados de Ribeirão Preto (Fuzii, 1989), Brasil em geral (Picanço, 1989) e Cuba em geral (Jordán, 1984) (Figura 3). Verifica-se semelhança de amplitude entre o intervalo de Barrinha com os do Brasil (Geral, Urbano e Rural) e de Cuba, porém com um deslocamento dos percentis 97 e 3 para idades mais baixas, na cidade de Barrinha. A semelhança entre amplitudes poderia ter como uma explicação a diversidade biológica (étnica), em al guns pontos semelhante entre as amostras. Cabe relembrar que os dados de Cuba e do Brasil se referem à década de 80, e ainda que os do Brasil foram colhidos entre mulheres de 10 a 20 anos (Picanço, 1989). Quando se compara a amplitude entre Barrinha e Ribeirão Preto, verifica-se que ela é nitidamente maior em Barrinha. Este dado poderia estar indicando as desigual dades sócio-econômicas entre as duas cidades.

Apesar de as medianas da IM estarem próximas entre as classes sociais 3 e 4, existe uma diferença no percentil 3, que é mais tardio na classe social 4, ou seja, naquela com piores condições, o que está de acordo com outros autores (Fuzii, 1989; Liestol \& Rosemberg, 1995). Pela grande amplitude de variação em relação aos percentis 3 e 97, de 5,36 anos na classe social 3 e 5,54 anos na classe 4, pode-se supor que a classe social interfere fortemente como componente de desigualdade na idade da menarca, além da grande diversidade genética que existe, de modo geral, em populações brasileiras.

Diferentemente do que se observa em outros trabalhos, neste estudo não foi encontrada diferença na IMM segundo o número de irmãos. Fuzii (1989) encontrou diferenças entre 0,16 a 0,33 anos quando comparou amostras de meninas com menos de dois irmãos, com dois e com mais de dois irmãos. Malina et al . (1997), estudando tamanho familiar e idade da menarca em atletas, observaram que a menarca era mais tardia entre 0,16 e 0,22 anos para cada irmão adicional.

A IMM mais precoce em Barrinha poderia ser em parte atribuída ao desemprego dos pais. A sua permanência mais tempo em casa, por um efeito feromonal (Burger \& Gochfeld, 1985), poderia antecipar a IM M. Porém, os resulta-
Figura 2

Distribuição em percentis da idade da menarca nas escolares do Município de Barrinha (1998) segundo variáveis sócio-econômicas.

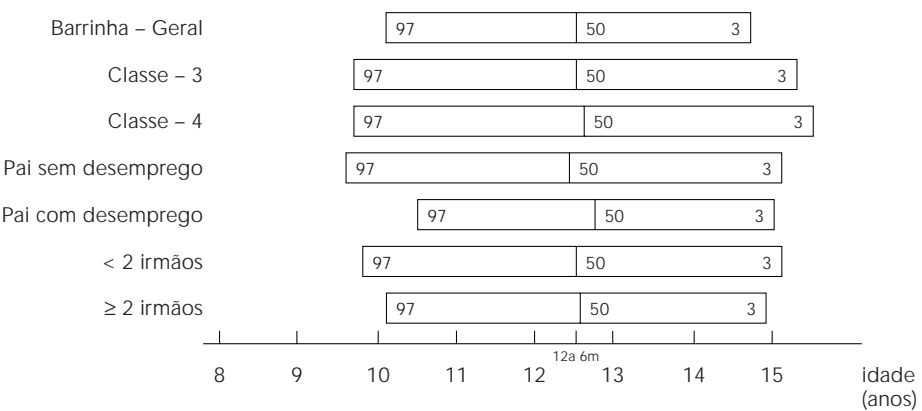

Figura 3

Distribuição em percentis da idade da menarca de escolares do Município de Barrinha (1998), comparada com dados do Brasil (1989), de Ribeirão Preto (1989) e de Cuba (1994).

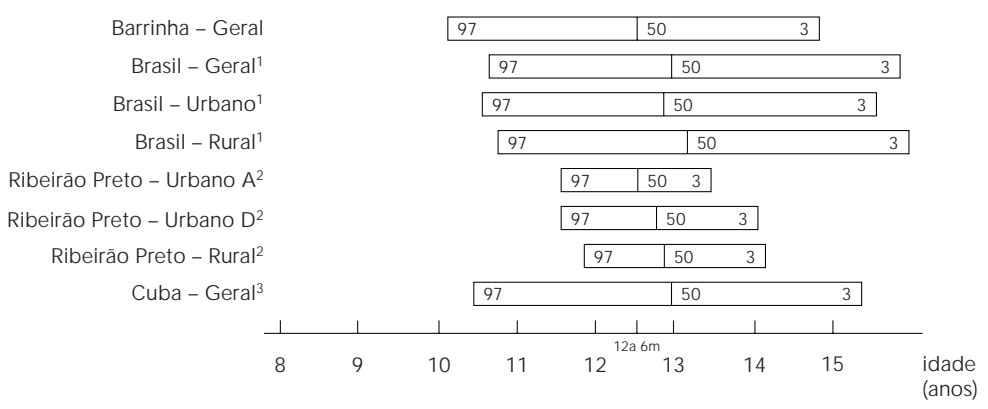

1Picanço (1989); 2Fuzii (1989); 3J ordán (1984).

dos indicam que o desemprego acompanhou o efeito relacionado às variáveis sócio-econômicas, mostrando um aumento da IMM nas filhas de pais com história de desemprego, o que concorda com os achados de Ulijaszek et al. (1991).

Quando se observa a amplitude do intervalo (dispersão) entre os percentis 3 e 97 de cada variável sócio-econômica, nota-se que essa amplitude não apenas é maior do que a amostra geral, como também a menarca ocorre em idades mais precoces, tanto quando se refere a número de irmãos menor que dois, quanto à situação de sem desemprego, quando comparadas com o grupo de situação com desemprego. Neste estudo, a amplitude de variação entre os 
percentis 3 e 97 foi de 5,14 anos para as meninas com menos de dois irmãos e 4,9 anos para as com dois ou mais irmãos. Fuzii (1989), estudando o número de irmãos, encontrou amplitudes de intervalos semelhantes nos grupos com menos de dois irmãos (2,14 anos), dois irmãos (2,22 anos) e com mais de dois irmãos (2,31 anos) em Ribeirão Preto. A diferença de amplitude de intervalo entre os estudos de Barrinha e Ribeirão Preto pode indicar uma maior heterogeneidade das amostras de Barrinha. Como pertencem a uma mesma região geoeconômica, com uma possível semelhança na diversidade étnica, as diferenças de amplitude ficariam mais por conta de fatores ambientais como os sócio-econômicos.

Ainda em relação à amplitude do intervalo, é importante observar que o percentil 97 em Barrinha se encontra abaixo dos de Ribeirão Preto, Brasil (diversas subamostras), e Cuba. Em geral isto indica que um número proporcionalmente maior de escolares em Barrinha está começando a menstruar mais cedo. Essa observação é importante quando se pensa em políticas de saúde que tenham como referência as adolescentes, na abordagem de temas como sexualidade, incluindo a menarca, gravi-

\section{Agradecimentos}

Às professoras Claudia Elena R. Piati, Dulcinei T. da S. Chagas, Gisele Regina R. Fuzato, Lucia Elena de O. Martins e Marta Cristina D. Pissal do, pela valiosa colaboração nas diversas fases do trabalho. À Professora Doutora Célia Pezzolo de Carvalho, do LEC/ FFCLRPUSP, por criar condições para o trabal ho conjunto. À Viviane Romeiro, pela confecção dos gráficos.

Este trabalho recebeu apoio financeiro do $\mathrm{CNPq}$ - Conselho Nacional de Desenvolvimento Científico e Tecnológico - e da FAEPA - Fundação de Apoio ao Ensino, Pesquisa e Assistência do Hospital das Clínicas da FMRP-USP. dez na adolescência, uso de métodos anticoncepcionais e aborto. A necessidade de políticas públicas voltadas especificamente para essa faixa etária é reforçada por dados recentes que mostram um aumento importante na gestação em adolescentes em Ribeirão Preto em 15 anos, de 14,1 para $17,5 \%$ (Ribeiro, 1999).

Em conclusão, a IMM entre escolares do Município de Barrinha em 1998 está abaixo de valores regionais e de países desenvolvidos. Mesmo que as condições de vida em Barrinha sejam compatíveis com padrões de zona rural, este indicador de maturação encontra-se dentro dos padrões de regiões urbanizadas. As diferenças encontradas na amplitude de variação entre os percentis 97 e 3 da IM segundo variáveis como classe social, situação de desemprego do pai e número de irmãos mostram que essa variação pode explicar em parte a diversidade biológica e refletir as desigualdades sócioeconômicas na cidade de Barrinha. Esses achados reforçam ainda a necessidade de se estudar a contribuição de outros fatores influentes na idade da menarca, bem como a necessidade de se implementar programas de saúde pública para adolescentes que contemplem o seu nível de maturidade.

\section{Referências}

BETTIOL, H.; BARBIERI, M. A.; GOMES, U. A.; ANDREA, M.; GOLDANI, M. \& RIBEIRO, E. R. O., 1998. Saúde perinatal: Metodologia e características da população estudada. Revista de Saúde Pública, 32:18-28.

BURGER, J. \& GOCHFELD, M. A., 1985. A hypothesis on the role of pheromones on age of menarch. Medical Hypothesis, 17:39-46.

CAMERON, N., 1998. Adulthood and developmental maturity. In: Human Growth and Development (S. J. Ulijaszek, F. E. Johnston \& M. A. Preece, eds.), pp. 233-235, Cambridge: Cambridge University Press.

CAMERON, N. \& NAGDEE, I., 1996. Menarcheal age in two generations of South African Indians. Annals of Human Biology, 23:113-119. 
COLLI, A. S.; CONCEIÇÃO, J. A. N. \& COELHO, H. S., 1985. Desenvolvimento pubertário em escolares de São Paulo. In: La Salud del Adolescente y el Joven en las Américas, OPAS Publicación Científica no 489, pp. 249-258, Washington, D.C.: Organización Panamericana de la Salud.

EVELETH, P. B., 1998. Menarche. In: Human Growth and Development (S. J. Ulijaszek, F. E. Johnston \& M. A. Preece, eds.), p. 228, Cambridge: Cambridge University Press.

FINNEY, D. J., 1964. Probit Analysis: A Statistical Treatment of the Sigmoid Response Curve. 2nd Ed., Cambridge: Cambridge University Press.

FUZII, H. H., 1989. Estudo Epidemiológico da Idade da Menarca no Município de Ribeirão Preto. Dissertação de Mestrado, Ribeirão Preto: Faculdade de Medicina de Ribeirão Preto, Universidade de São Paulo.

GRABER, J. A.; BROOKS-GUNN, J. \& WARREN, M. P., 1995. The antecedents of menarcheal age: Heredity, family environment, and stressful life events. Child Development, 66:346-359.

GREKSA, L. P., 1990. Age of menarche in Bolivian girls and Aymara ancestry. Annals of Human Biology, 17:49-53.

HEALY, M. J. R., 1986. Statistics of growth standards. In: Human Growth (F. Falkner \& J. M. Tanner, eds.), v. 2, pp. 47-58, New York: Plenum.

HELM, P. \& GRONLUND, L., 1998. A halt in the secular trend towards earlier menarche in Denmark. Acta Obstetricia et Gynecologica Scandinavica, 77:198-200.

HERM AN-GIDDENS, M. E.; SLORA, E. J.; WASSERMAN, R. C.; BOURDONY, C. J.; BHAPKAR, M. V.; KOCH, G. G. \& HASEMEIER, C. M., 1997. Secondary sexual characteristics and menses in young girls seen in office practice: A study from the pediatric research in office settings network. Pediatrics, 99:505-512.

IBGE (Fundação Instituto Brasileiro de Geografia e Estatística), 1996. Censo 1996, Estimativa para 1998. Ribeirão Preto: Direção Regional de Saúde XVIII, Ministério da Saúde.

JORDÁN, J. R., 1984. Desarrollo Humano en Cuba. Ciudad de la Habana: Fondo de las Naciones Unidas para la Infancia.

LIESTOL, K. \& ROSENBERG, M., 1995. Height, weight and menarcheal age of schoolgirls in Oslo - An update. Annals of Human Biology, 22:199-205.

MAC MAHON, B., 1973. Age at menarche. Vital Health Statistics, 11:1-28.

MALINA, R. M.; KATZM ARZYK, P. T.; BONCI, C. M .; RYAN, R. C. \& WELLENS, R. E., 1997. Family size and age at menarche in athletes. Medicine Science in Sports and Exercise, 29:99-106.
MARSHALL, W. A. \& TANNER, J. M., 1986. Puberty. In: Human Growth (F. Falkner \&J. M. Tanner, eds.), v. 2, pp. 171-203, New York: Plenum.

OLSEN, J. \& FRISCHE, G., 1973. Social differences in reproductive health. Scandinavian Journal of Social Medicine, 21:90-97.

OHSAWA, S.; JI, C.-Y. \& KASAI, N., 1997. Age at menarche and comparison of the growth and performance of pre- and post- menarcheal girls in China. American Journal of Human Biology, 9:205212.

PICANÇO, M. R. A., 1989. A Idade da Menarca da Menina Brasileira: Os Fatores Sócio-econômicose as Diferenças Regionais. Dissertação de Mestrado, Rio de Janeiro: Instituto Fernandes Figueira, Fundação Oswaldo Cruz.

RIBEIRO, E. R. O., 1999. Saúde Perinatal em Ribeirão Preto: Comparações entre duas Coortes de Mães Adolescentes. Tese de Doutorado, Ribeirão Preto: Faculdade de Medicina de Ribeirão Preto, Universidade de São Paulo.

ROBERTS, D. F. \& DANN, T. C., 1975. A 12 - year study of menarcheal age. British Journal of Preventive and Social Medicine, 29:31-39.

TANNER, J. M., 1989. Puberty. In: Foetus Into Man (J. M. Tanner, ed.), pp. 58-74, Ware: Castlemead Publications.

THIOLLENT, M., 1988. Metodologia da PesquisaAção. São Paulo: Cortez Editora.

ULIJASZEK, S. J.; EVANS, E. \& MILLER, D. S., 1991. Age at menarche of European, Afro-Caribbean and Indo-Pakistani schoolgirls living in London. Annals of Human Biology, 18:167-175.

VALENZUELA, C. Y.; NUÑEZ, E. \& TAPIA, C., 1991. Month at menarche: A re-evaluation of the seasonal hypothesis. Annals of Human Biology, 18:383-393.

VAN WIERINGEN, J. C., 1986. Secular Growth Changes. In: Human Growth (F. Faulkner \& J. M. Tanner, eds.), v. 3, pp. 307-331. New York: Plenum Press.

VERCAUTEREN, M. \& SUZANNE, C., 1985. The secular trend of height and menarche in Belgium: Are there any signs of a future stop? European Journal of Pediatrics, 144:306-309.

VERONESI, F. M. \& GUERESI, P., 1994. Trend in menarcheal and socioeconomic influence in Bologna (Northern Italy). Annals of Human Biology, 21: 187-196.

WELLENS, R.; MALINA, R. M.; BEUNEN, G. \& LEFEVRE, J., 1990. Age at menarche in Flemish girls: Current status and secular change in the 20th century. Annals of Human Biology, 17:145-152. 